Gazi University
Journal of Science
http://dergipark.gov.tr/gujs

\title{
Quantum dot Cellular Automata based Fault Tolerant Fingerprint Authentication Systems using Reversible Logic Gates
}

\author{
Suhaib AHMED ${ }^{1 *}$ (D), Syed Farah NAZ ${ }^{2}$ (D) Sparsh SHARMA $^{3}$ \\ ${ }^{1}$ Baba Ghulam Shah Badshah University, Department of Electronics and Communication Engineering, 185234, Rajouri, Jammu and Kashmir, \\ India \\ ${ }^{2}$ Indian Institute of Technology, Discipline of Electrical Engineering, 181221, Jammu, Jammu and Kashmir, India \\ ${ }^{3}$ Baba Ghulam Shah Badshah University, Department of Computer Science and Engineering Engineering, 185234, Rajouri, Jammu and \\ Kashmir, India
}

Highlights

- Fault tolerance analysis of various XOR gates against single cell addition and missing defects.

- Highly fault tolerant XOR is used to design four Fingerprint Authentication Systems in QCA.

- Energy dissipation analysis of all four FAS designs is presented at different kink energy levels.

- The proposed FAS designs are highly area and cost efficient.

Article Info

Received: 20 Sep 2020

Accepted: 15 Apr 2021

Keywords

$Q C A$

Fault tolerant design

Reversible logic

Nanoelectronics

Biometric system

\begin{abstract}
The limits and difficulties looked by CMOS innovation in the nano system has prompted the exploration of other potential advancements which can work with same functionalities anyway with lower power scattering and higher speed. One such technology is Quantum dot Cellular Automata (QCA). In this paper, QCA is explored to design the authentication system. This paper first presents the basic operating principle of a Fingerprint Authentication System (FAS) followed by fault tolerance analysis of four efficient XOR gate designs in the literature. The XOR gate is then used in the proposed four fault tolerant designs of reversible FAS in QCA, which are based on different reversible gates. Based on the evaluation of different performance parameters, it is seen that the proposed FAS designs are cost efficient and achieve improvement up to $59.46 \%$ in terms of number of cells, $67.16 \%$ improvement in cell area, $67.14 \%$ improvement in total area, $66.67 \%$ improvement in latency and $90.51 \%$ improvement in terms of circuit cost from the existing design Furthermore, the energy dissipation examination of the proposed designs is also additionally introduced. Subsequently, the proposed designs can be effectively used in biometric applications demanding ultra-low power consumption, higher operating speed and minimal area utilization.
\end{abstract}

\section{INTRODUCTION}

The fundamental nanometer-scale physical constraints of complementary metal oxide semiconductor (CMOS) technology are nearing. It causes a slew of short-channel effects and contributes to the deterioration of field-effect transistor characteristics [1-3]. Alternative nanotechnologies, such as the nanowire transistor, quantum-dot cellular automata (QCA), carbon nanotube field-effect transistor, and others, have already been offered solutions to these problems. QCA, first proposed by C. S. Lent in [1], has been identified as the most recent powerless technology capable of replacing nanoscale-based transistorbased semiconductor technology. It offers intriguing benefits such as increased device density and greater working switching frequencies (in the range of a few THz) at the nanoscale. Because the energy dissipation during state transition and propagation in QCA is negligible [4-6], there is significantly minimal energy dissipation when compared to CMOS technology. QCA is thoroughly investigated, and many logics are being proposed for diverse purposes, including reversible logic [6-9], arithmetic circuits [4, 5, 10-16], code converters [17-21], sequential circuits [17, 22-25], memories [26-29], and so on. 


\subsection{QCA Preliminaries}

- QCA Cell: It comprises four quantum wells, two of which have two electrons in diagonally opposing locations to achieve the lowest electronic repulsion, resulting in two binary states with information [30-32]. As seen in Figure 1, one of the electron locations describes '1' while the other describes '0.' Figure 2 shows the cells forming a QCA wire in tandem [3, 33].

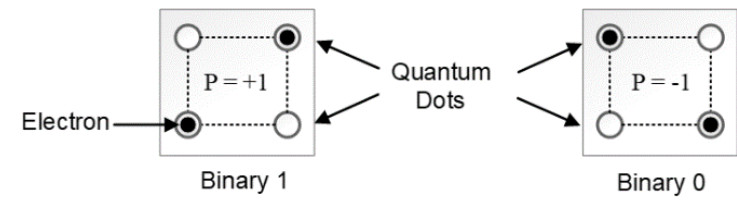

Figure 1. QCA cell and its polarizations

\section{IN}

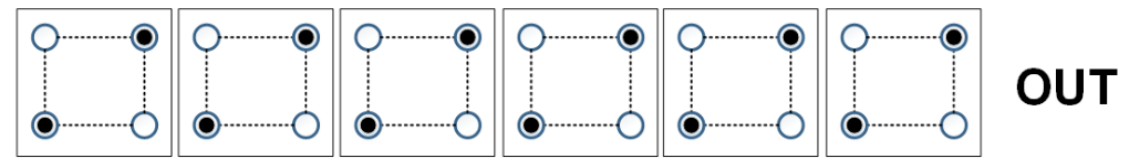

Figure 2. QCA Binary wire

- QCA Logic Gates: As shown in Figure 3(a), the basic logic gate in QCA is a three-input majority voter gate $[3,9]$. The $\mathrm{M}(\mathrm{A}, \mathrm{B}, \mathrm{C})=\mathrm{AB}+\mathrm{BC}+\mathrm{CA}$ mathematical operation is used to operate the gate. Figure 3(b) and Figure 3(c) illustrate the output of an OR gate when one of the inputs is set to ' 1 ' and the output of an AND gate when one of the inputs is set to ' 0 '. (c). Inverter, besides being the basic building block of the Majority Voter, is used to invert the input logic. Figure $4[3,9]$ shows various inverter setups in QCA.

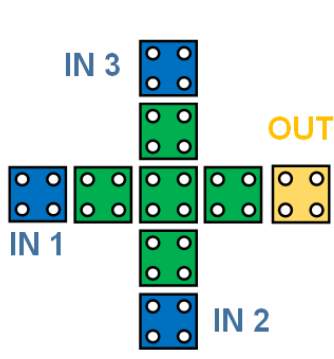

(a)

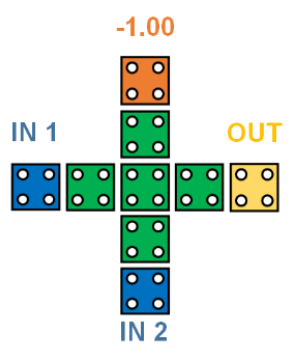

(b)

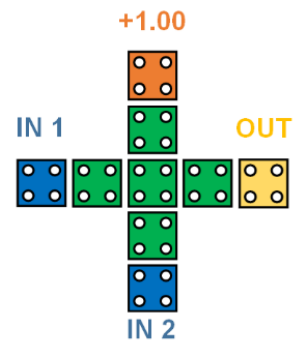

(c)

Figure 3. Design of (a) 3-Input majority voter gate (b) Majority voter based AND gate and (c) Majority voter based OR gate in QCA [3, 9]

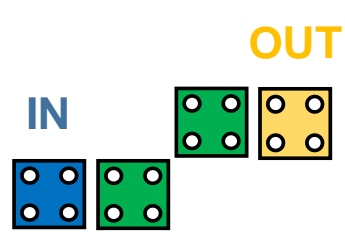

(a)

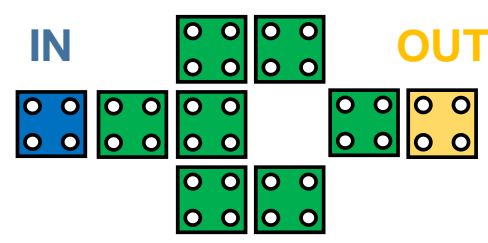

(b)

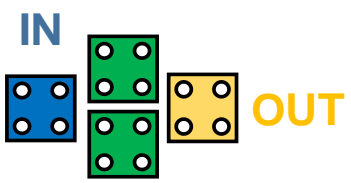

(c)

Figure 4. Various configurations of QCA Inverters (a) Half-cell displaced, (b) Large robust and (c) Rotated cell [3, 9]

- Clocking in QCA: A four-phase clock is used in QCA circuits [34], as shown in Figure 5 [11]. The QCA cells are unpolarized at first, resulting in a low potential. During the switch phase, the polarization of a QCA cell is mainly controlled by the polarization of nearby cells, and the potential of the electrons begins to climb. Furthermore, once the electron reaches its maximum potential 
vitality at the end of the switching period, no state transition happens. In the hold phase, the cell maintains its initial state and maintains a high level of potential vitality. The potential liveliness of the electrons begins to decrease during the release and relax phase, eventually returning to null polarization $[3,12,33]$.

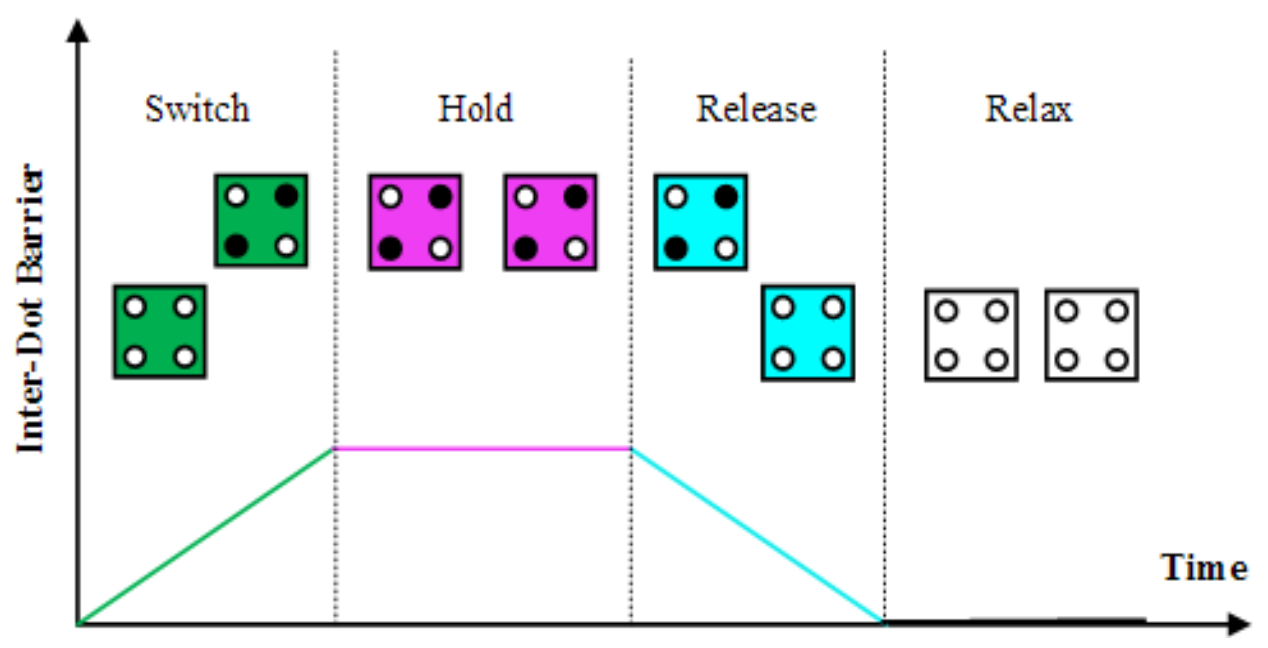

Figure 5. Illustration of clocking in QCA

The organization of the remaining paper is as follows: the brief description of the Fingerprint Authentication System (FAS) and its basic operation along with the review on existing design of FAS in QCA is given in Section 2. Various XOR gates and their fault tolerance analysis against single cell addition and missing defects is presented in section 3 along with their performance comparison followed by four proposed QCA designs of FAS in section 4. The energy dissipation analysis of all proposed FAS designs is presented in section 5. The comparison of various performance metrics is given in section 6 followed by concluding remarks in section 7 .

\section{FINGERPRINT AUTHENTICATION SYSTEM}

The fingerprint authentication systems (FAS), based on digital logic and represented in in Figure 6, is utilized to coordinate with the information unique mark picture with existing pictures accessible in the data set [35]. On the off chance that match exists, the client is verified. All bits of the info picture (input) are checked consecutively with the pictures in the data set and when the match occurs, the comparing yield bit is set to rationale ' 1 '. On the off chance that toward the finish of coordinating, every one of the pieces of yield are '1' then the match between these two images exists [35]. This mechanism can be perceived from Table 1.

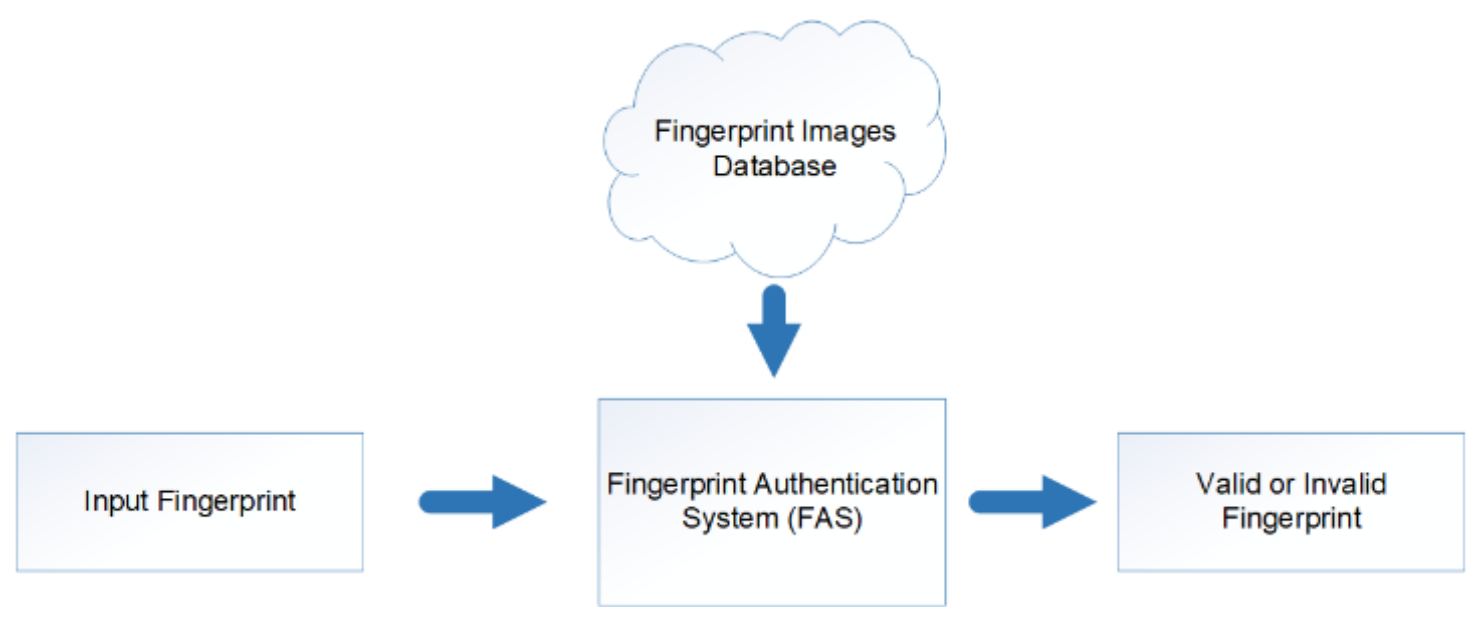

Figure 6. FAS conceptual block diagram [35] 
Table 1. Operating Conditions of FAS

\begin{tabular}{|c|c|c|}
\hline $\mathbf{A}$ & B & Output \\
\hline "-1" or " Logic 0" & "-1" or "Logic 0" & $"+1 "$ or "Logic 1" \\
\hline "-1" or " Logic 0" & $"+1 "$ or " Logic 1" & $"-1 "$ or " Logic $0 "$ \\
\hline$"+1 "$ or " Logic 1" & "-1" or "Logic 0" & "-1" or " Logic 0" \\
\hline$"+1 "$ or " Logic 1" & $"+1 "$ or " Logic 1" & $"+1 "$ or "Logic 1" \\
\hline
\end{tabular}

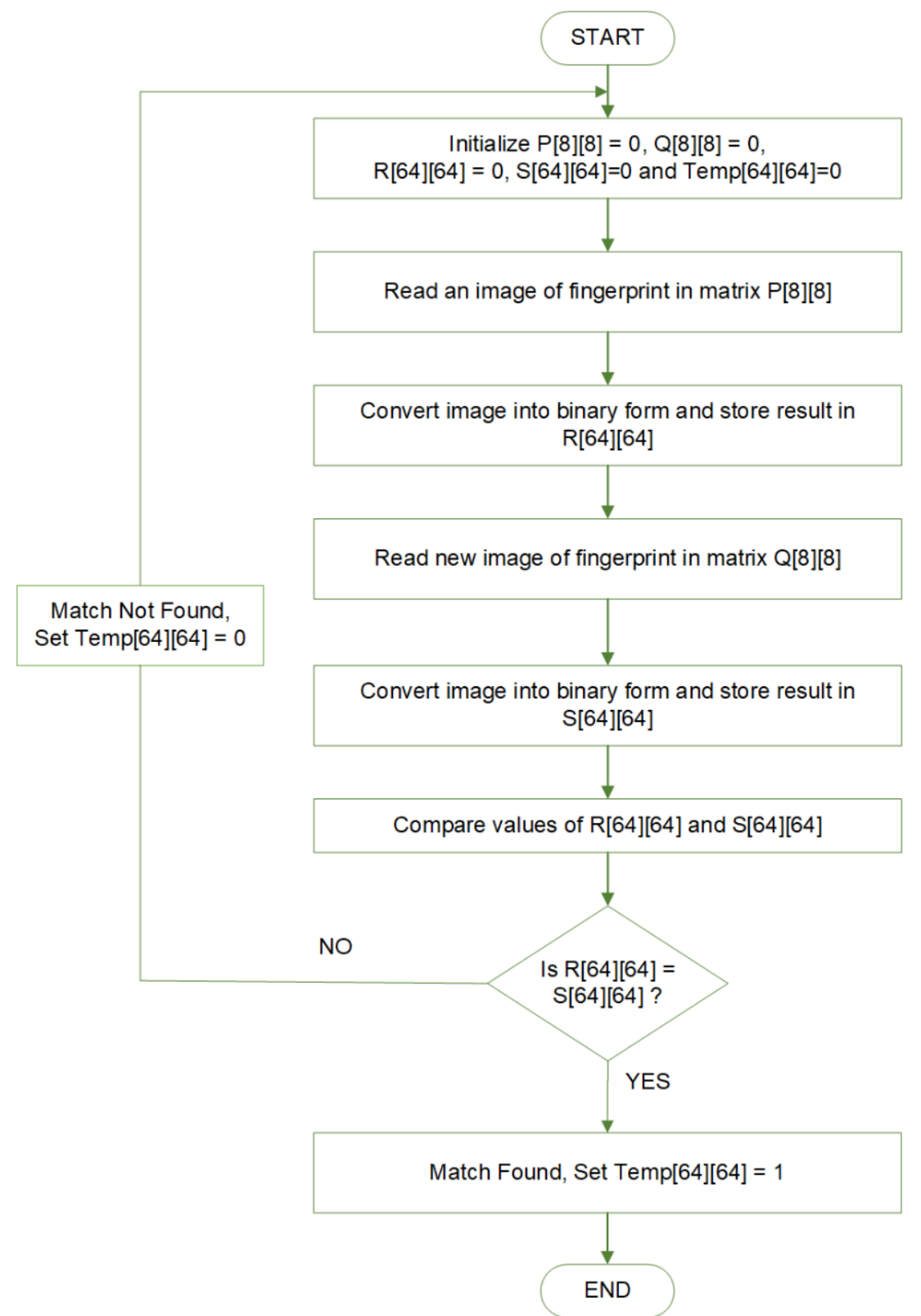

Figure 7. Operating steps of FAS [35]

It is seen from Table 1 that FAS operation is similar to that of an XNOR gate, hence the equations of FAS can be written as:

$Y=\bar{A} \bar{B}+A B$

This operation can be visualized using the operating mechanism depicted in Figure 7. One such fingerprint authenticator (FPA) has been presented in [36], using a $2 \times 2$ Feynman Gate (shown in Figure 8). 


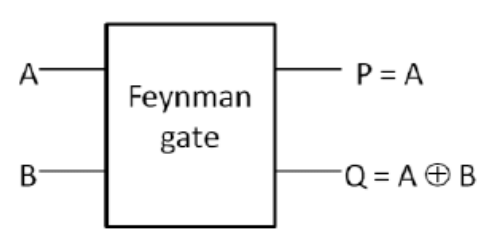

Figure 8. Feynman Gate

Debnath et al. in [36] presented an FPA by modifying the input A (written as X) to A' (written as X') of the Feynman gate as depicted in Figure 9.

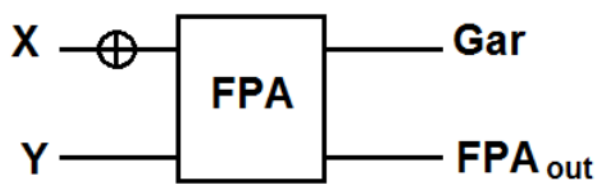

Figure 9. FPA proposed in [36]

The QCA implementation of this FPA, shown in Figure 10, by Debnath et al. in [36] was done using the equations based on the majority voter concept

$$
\begin{aligned}
& G A R=\bar{X} \\
& F P A_{\text {out }}=M(M(\bar{X}, \bar{Y}, 0), M(X, Y, 0), 1) .
\end{aligned}
$$

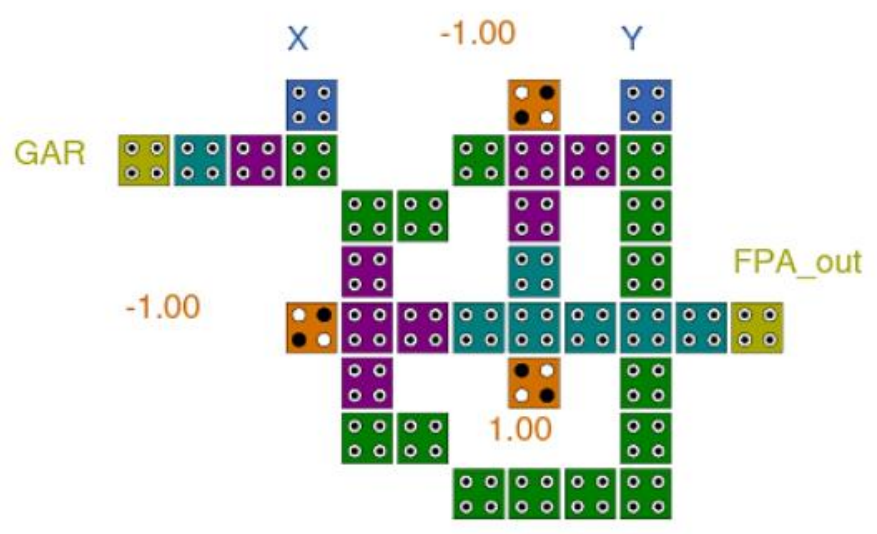

Figure 10. QCA based FPA proposed in [36]

\section{XOR GATES IN QCA}

The basic operating principle of a Fingerprint Authentication System (FAS) is identical to the operation of XNOR gate and XNOR gate can be directly designed from XOR gate. Hence, in this section, various efficient XOR gates proposed in the literature have been presented. In addition to this, since the application in concern requires accuracy, so a fault tolerant design is of prime importance. To achieve this, fault tolerance analysis of these XOR gates has also been presented in order to select the most efficient and fault tolerant design of XOR gate for the proposed FAS.

\subsection{Design - 1}

The first design considered in this paper is of Majeed et al. in [37] who proposed an XOR gate shown in Figure 11(a). It consists of 9 cells having 0.25 clock cycle latency and cell area $0.003 \mu \mathrm{m}^{2}$. The total area occupied by this design is $0.01 \mu \mathrm{m}^{2}$. The gird diagram used for analyzing the fault tolerance of this gate is shown in Figure 11(b). 


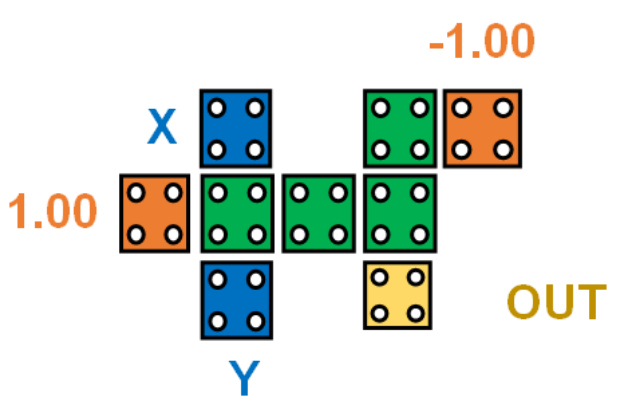

(a)

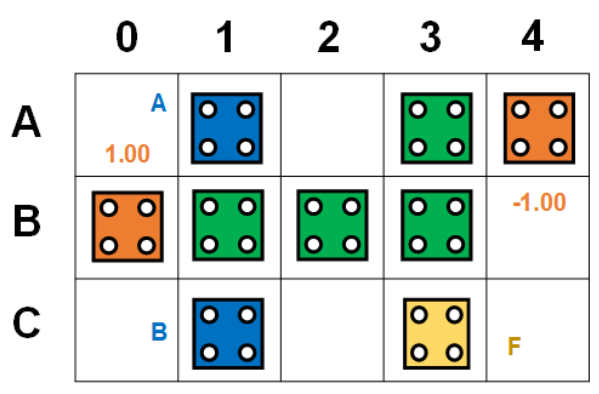

(b)

Figure 11. (a) XOR gate proposed in [37] (b) Grid diagram of XOR gate

\subsection{Design - 2}

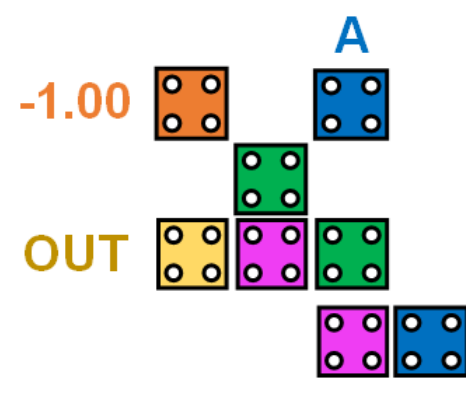

(a)

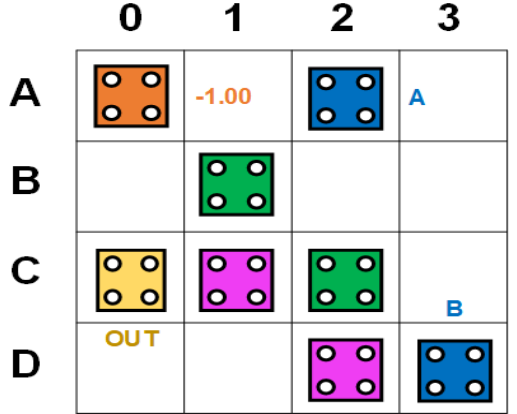

(b)

Figure 12. (a) XOR gate used in [12] and (b) Grid diagram of XOR gate

Another XOR gate considered in this paper is shown in Figure 12(a). This design occupies cell area of $0.0026 \mu \mathrm{m}^{2}$ and is designed with 8 cells having 0.5 clock cycle latency. The total area occupied by this design is $0.0061 \mu \mathrm{m}^{2}$. The gird diagram used for analyzing the fault tolerance of this gate is shown in Figure 12(b). There are two cases available here viz. Case 1 and Case 2. In Case 1, the clocking of additional cell D1 is in clock zone 1 whereas in Case2, the clocking of D1 is in clock zone 2.

\subsection{Design -3}

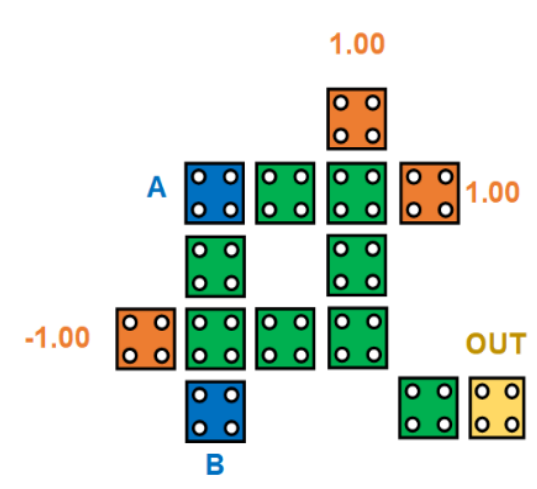

(a)

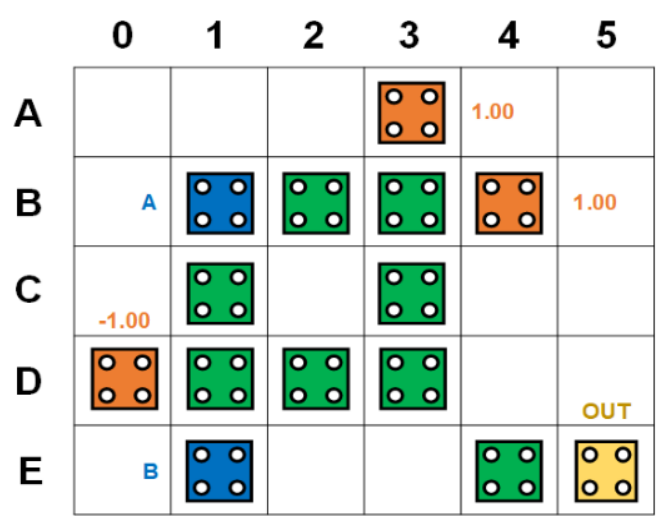

(b)

Figure 13. (a) XOR gate proposed in [38] and (b) Grid diagram of XOR gate

The third XOR gate considered in this paper is shown in Figure 13(a). This design occupies cell area of $0.0045 \mu \mathrm{m}^{2}$ and is designed with 14 cells having 0.25 clock cycle latency. The total area occupied by this design is $0.0116 \mu \mathrm{m}^{2}$. The gird diagram used for analyzing the fault tolerance of this gate is shown in Figure 13(b). 


\subsection{Design - 4}

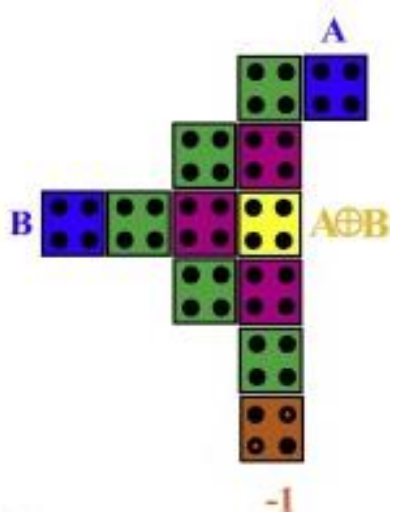

(a)

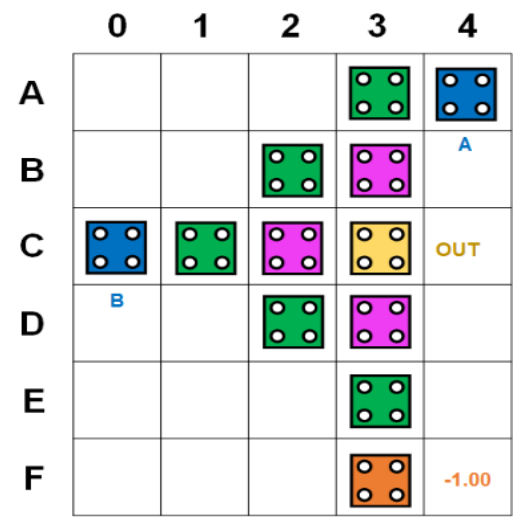

(b)

Figure 14. (a) XOR gate proposed in [39] and (b) Grid diagram of XOR gate

The fourth XOR gate considered in this paper is shown in Figure 14(a). This design occupies cell area of $0.0039 \mu \mathrm{m}^{2}$ and is designed with 12 cells having 0.5 clock cycle latency. The total area occupied by this design is $0.0116 \mu \mathrm{m}^{2}$. The gird diagram used for analyzing the fault tolerance of this gate is shown in Figure 14(b).

\subsection{Performance Comparison of Fault Tolerance of XOR Gates}

Table 2. Fault tolerance performance comparison

\begin{tabular}{|c|c|c|c|c|}
\hline \multicolumn{2}{|c|}{ XOR Design No. } & $\begin{array}{c}\text { Fault Tolerance } \\
\text { against Single Cell } \\
\text { Addition Defect }\end{array}$ & $\begin{array}{c}\text { Fault Tolerance } \\
\text { against Single Missing } \\
\text { Cell Defect }\end{array}$ & $\begin{array}{c}\text { Overall Fault } \\
\text { Tolerance }\end{array}$ \\
\hline \multirow{2}{*}{2} & $70.83 \%$ & $43.75 \%$ & $60 \%$ \\
\hline \multirow{2}{*}{2} & Case 1 & $69.44 \%$ & $50 \%$ & $63.46 \%$ \\
\cline { 2 - 3 } & Case 2 & $63.89 \%$ & $62.5 \%$ & $59.62 \%$ \\
\hline \multirow{2}{*}{3} & $84.375 \%$ & $53.125 \%$ & $77.08 \%$ \\
\hline \multirow{2}{*}{4} & $82.14 \%$ & & $71.59 \%$ \\
\hline
\end{tabular}

The fault tolerance analysis of these designs against single cell addition and missing defects was performed using the mechanism presented in [40]. All these designs have been designed using QCADesginer tool [41]. Based on the fault tolerance comparison of various XOR designs presented in Table 2, it is observed that Design-3 has highest overall fault tolerance against single cell addition and omission defects and hence this design will be used for designing the Fingerprint Authentication System.

\section{PROPOSED FINGERPRINT AUTHENTICATION SYSTEMS (FAS) IN QCA}

In this section four different designs of Fingerprint Authentication System (FAS) are proposed using different reversible logic gates.

\subsection{Design - 1 (Using Feynman Gate)}

Feynman gate [42] is a $2 \times 2$ reversible gate having the following output equations:

$P=A$

$Q=A \oplus B$. 


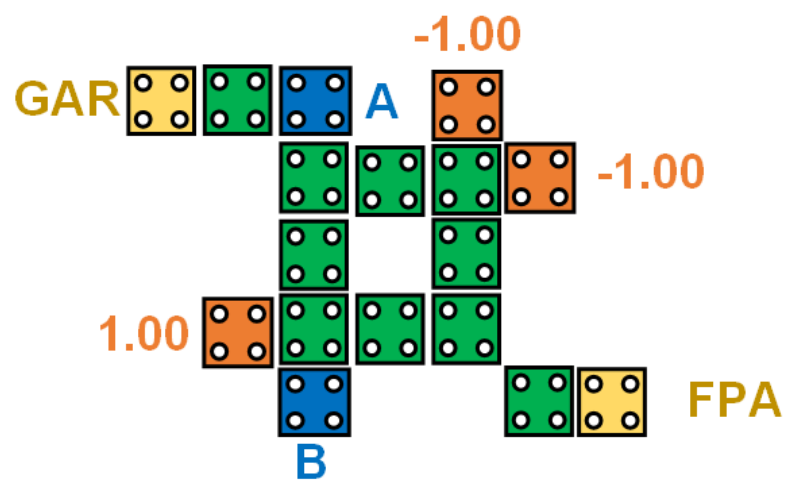

Figure 15. Proposed Design-1 of FAS using Feynman Gate

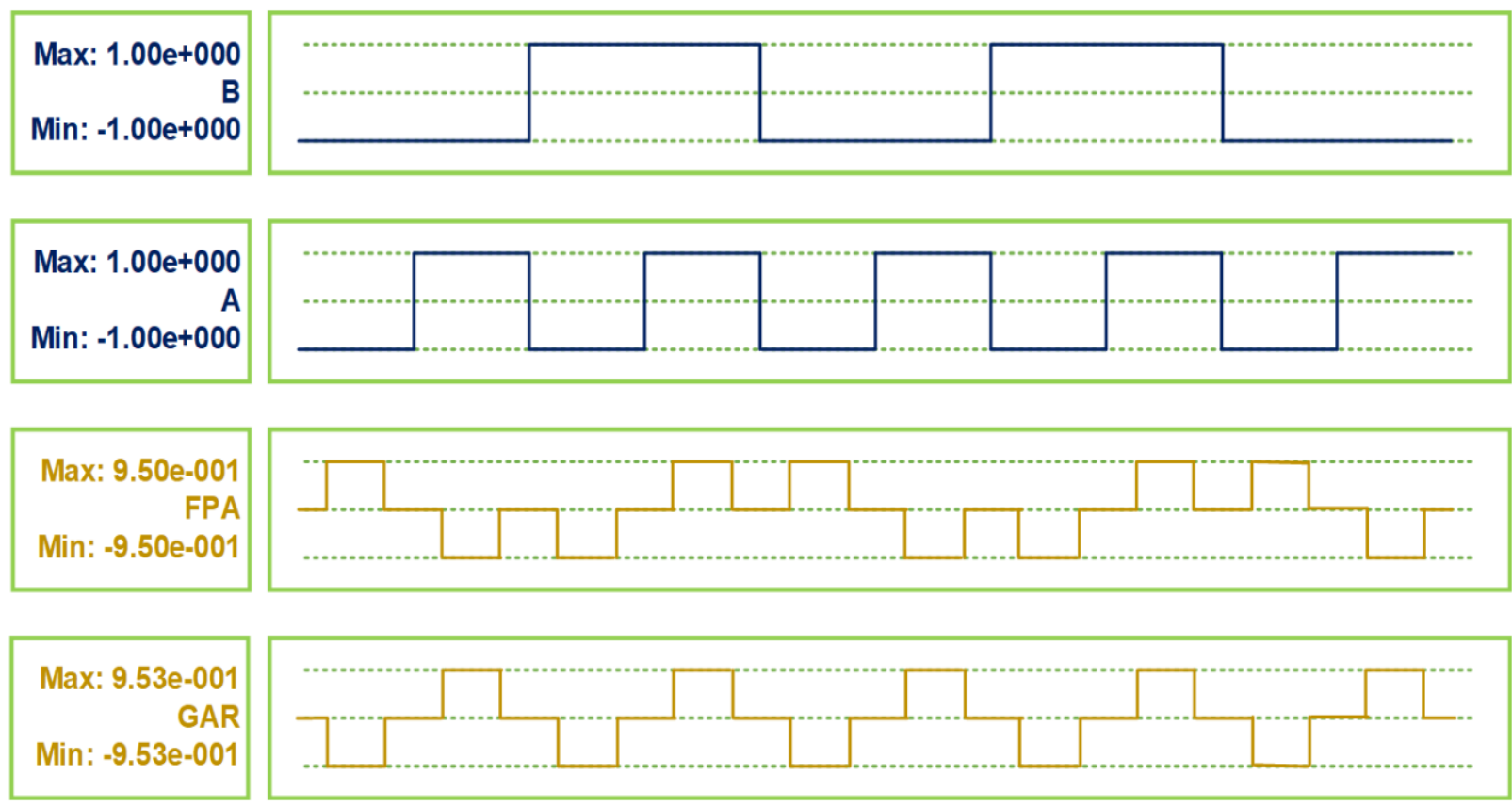

Figure 16. Input and output waveforms of proposed QCA design-1 of FPA

The above equations have been implemented using fault tolerant XOR gate (Design-3) and an inverter has been applied at the output Q to obtain the desired FPA output. The proposed implementation in QCA is shown in Figure 15. This design occupies cell area of $0.00486 \mu \mathrm{m}^{2}$ and is designed with 15 cells having 0.25 clock cycle latency. The total area occupied by this design is $0.011564 \mu \mathrm{m}^{2}$. The design can be validated from the simulation input and output waveforms shown in Figure 16 obtained from QCADesigner tool [41].

\subsection{Design - 2 (Using BJN Gate)}

BJN gate [43] is a $3 \times 3$ reversible gate having the following output equations:

$P=A$

$Q=B$

$R=(A+B) \oplus C$.

If either input B or input A is set to ' 0 ' and an inverter is applied at the output $\mathrm{R}$, we can obtain the desired FPA output. The proposed implementation in QCA using fault tolerant XOR gate, when input B is set to ' 0 ', is shown in Figure 17. This design occupies cell area of $0.007776 \mu \mathrm{m}^{2}$ and is designed with 24 cells having 0.5 clock cycle latency. The total area occupied by this design is $0.031284 \mu \mathrm{m}^{2}$. The design can be 
validated from the simulation input and output waveforms shown in Figure 18 obtained from QCADesigner tool [41].

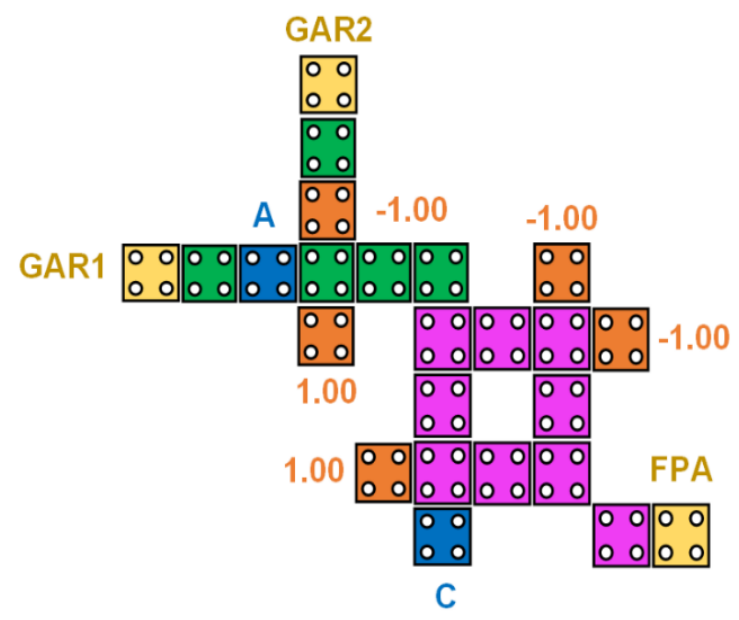

Figure 17. Proposed Design-2 of FAS using BJN Gate

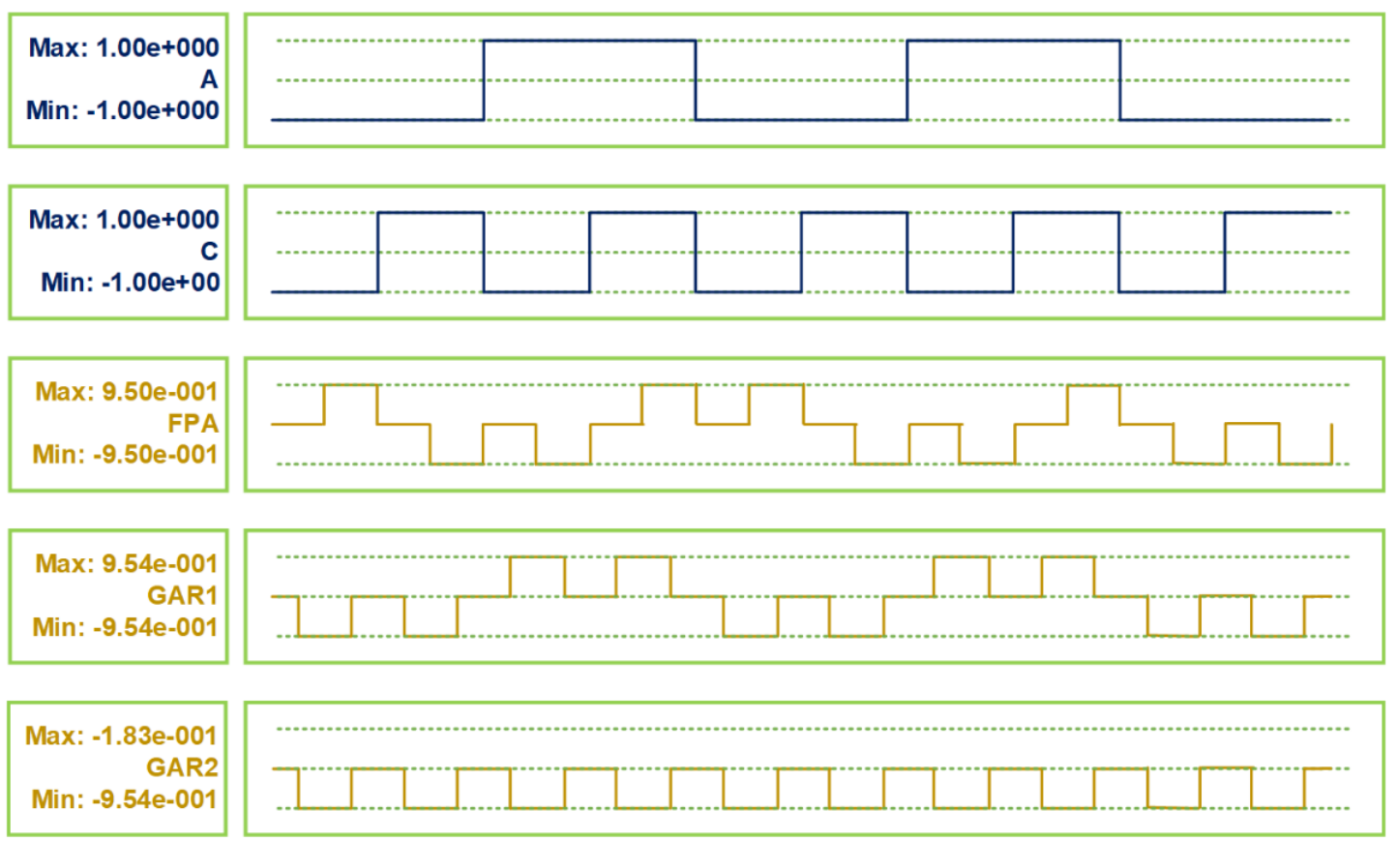

Figure 18. Input and output waveforms of proposed QCA design-2 of FAS

\subsection{Design - 3 (Using Toffoli Gate)}

Toffoli gate [44] is a $3 \times 3$ reversible gate having the following output equations:

$P=A$

$Q=B$

$R=A B \oplus C$.

If either input A or input B is set to ' 1 ' and an inverter is applied at the output $R$, we can obtain the desired FPA output. The proposed implementation in QCA using fault tolerant XOR gate, when input B is set to ' 1 ', is shown in Figure 19. This design occupies cell area of $0.008748 \mu \mathrm{m}^{2}$ and is designed with 27 cells having 0.5 clock cycle latency. The total area occupied by this design is $0.024564 \mu \mathrm{m}^{2}$. The design can be 
validated from the simulation input and output waveforms shown in Figure 20 obtained from QCADesigner tool [41].

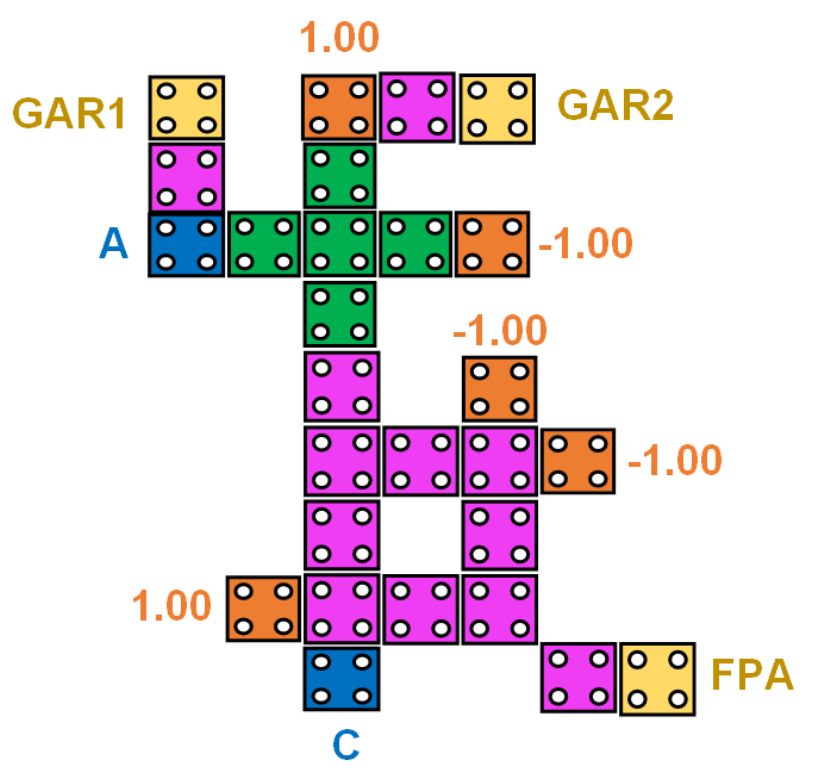

Figure 19. Proposed Design-3 of FAS using Toffoli Gate

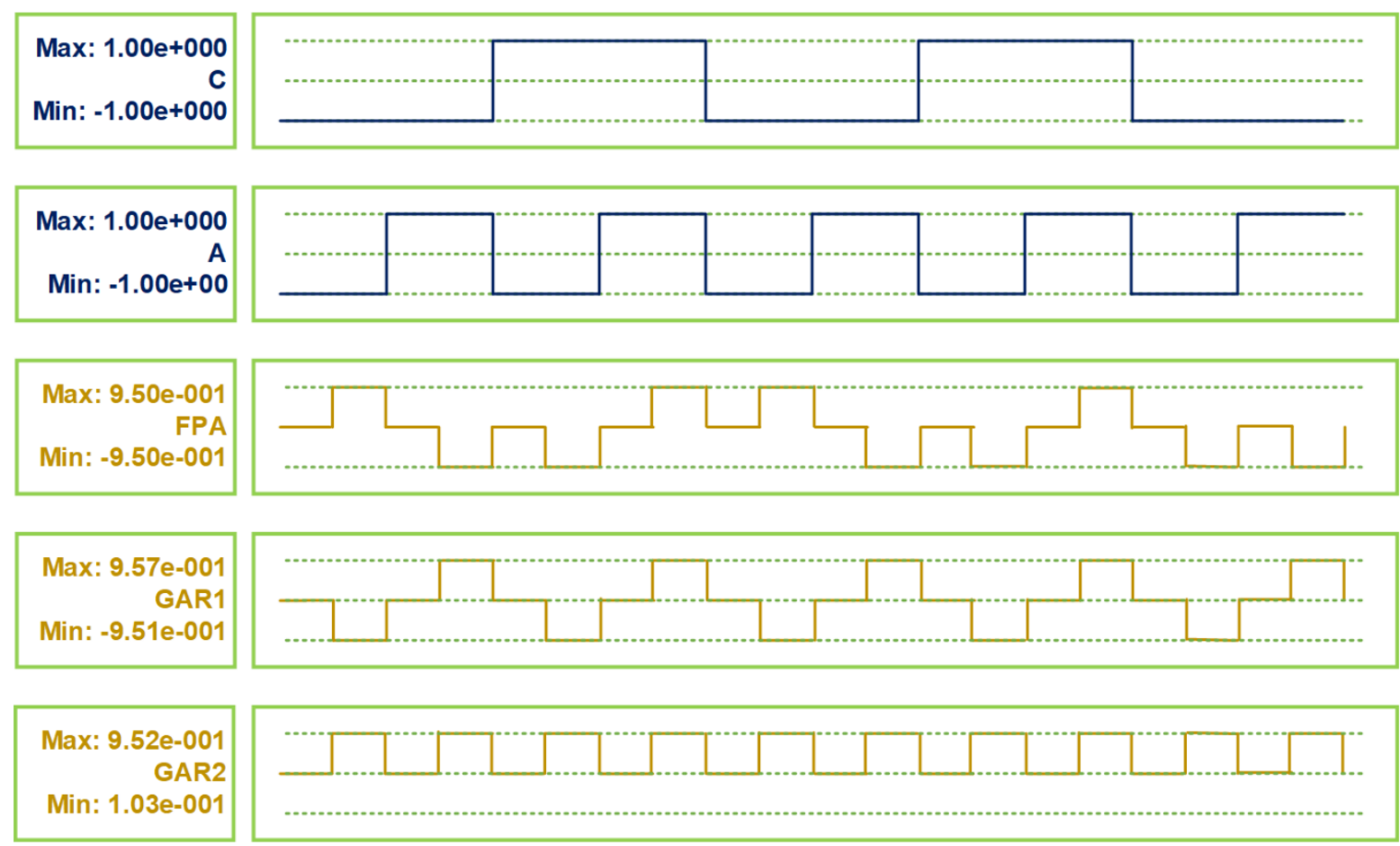

Figure 20. Input and output waveforms of proposed QCA design-3 of FAS

\subsection{Design - 4 (Using Double Feynman Gate)}

Double Feynman gate [45] is a 3x3 reversible gate having the following output equations:

$$
\begin{aligned}
& P=A \\
& Q=A \oplus B \\
& R=A \oplus C .
\end{aligned}
$$


As is evident from the equations, if an inverter is applied at outputs Q and R, we can get two XNOR gates from a single gate. This will lead to an additional functionality of FAS as the proposed system will be able to detect two different fingerprints simultaneously. The proposed implementation in QCA, using fault tolerant XOR gate, is shown in Figure 21. This design occupies cell area of $0.010368 \mu \mathrm{m}^{2}$ and is designed with 32 cells having 0.25 clock cycle latency. The total area occupied by this design is $0.030084 \mu \mathrm{m}^{2}$. The design can be validated from the simulation input and output waveforms shown in Figure 22 obtained from QCADesigner tool [41].

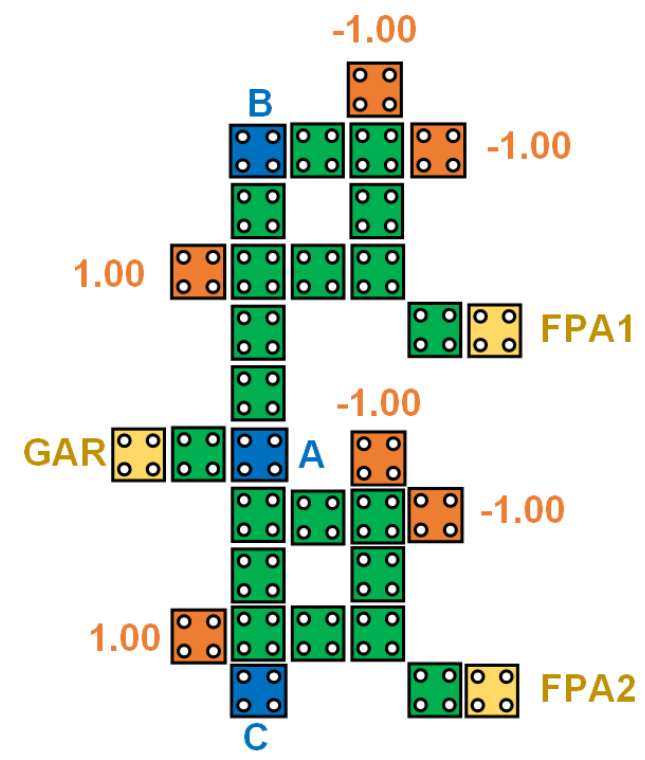

Figure 21. Proposed Design-4 of FAS using Double Feynman Gate

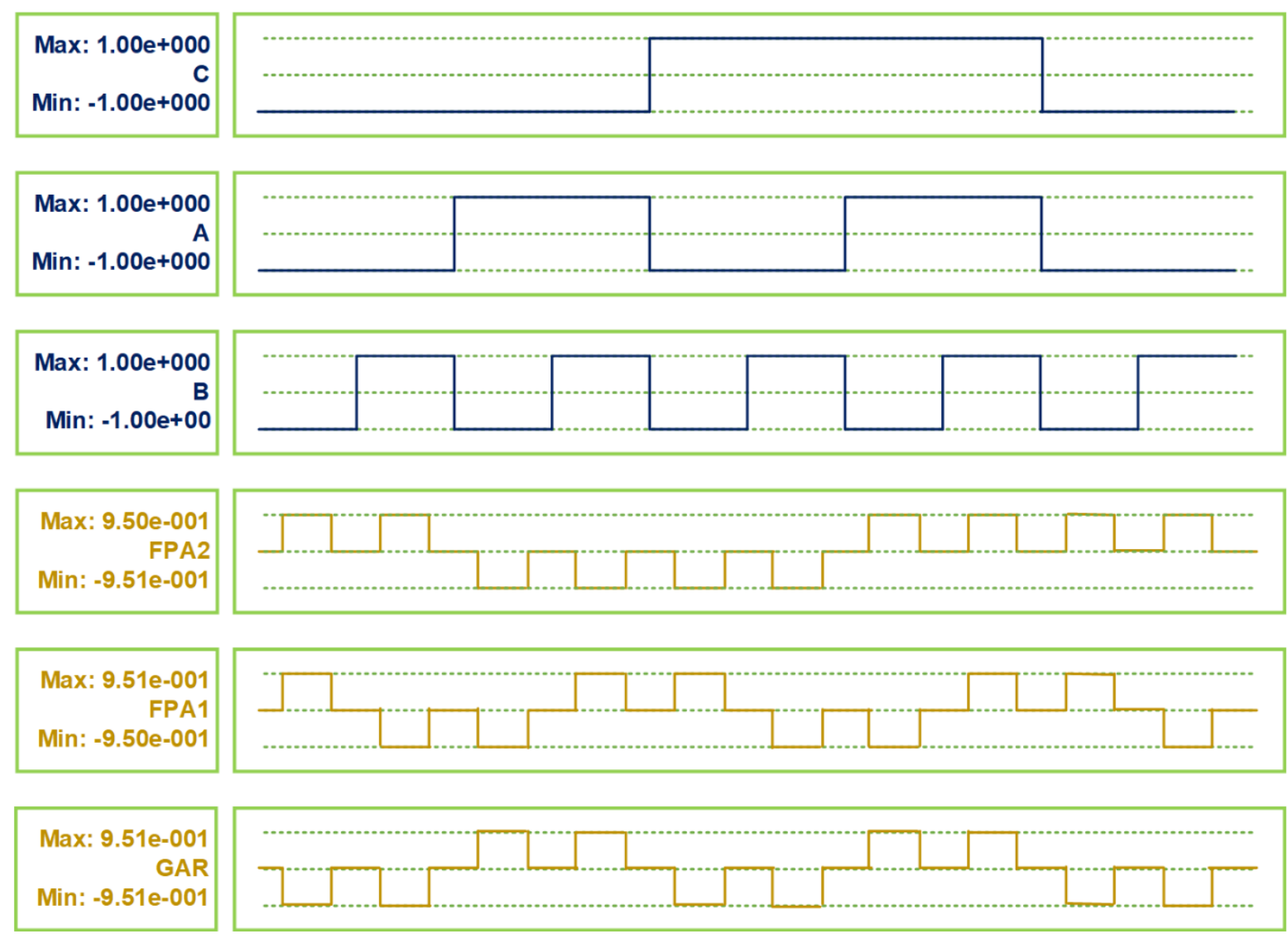

Figure 22. Input and output waveforms of proposed QCA design-4 of FAS 


\section{ENERGY DISSIPATION ANALYSIS}

The energy dissipation evaluation of the FAS designs proposed in this paper has been performed using QCAPro tool [46]. This tool calculates the Hamiltonian, given in Equation (15), for energy calculations by using Hatree-Fork $[47,48]$ method. It is a probabilistic approximation method for energy calculation.

$H=\left[\begin{array}{cc}\frac{-E_{k}}{2} \sum_{i} C_{i} f_{i, j} & -\gamma \\ -\gamma & \frac{E_{k}}{2} \sum_{i} C_{i} f_{i, j}\end{array}\right]=\left[\begin{array}{cc}\frac{-E_{k}}{2}\left(C_{j-1}+C_{j+1}\right) & -\gamma \\ -\gamma & \frac{E_{k}}{2}\left(C_{j-1}+C_{j+1}\right)\end{array}\right]$

The per clock cycle $\left(\mathrm{T}_{\mathrm{cc}}\right)$ energy dissipated per clock cycle is then calculated using the Hamiltonian, evaluated in Equation (15) along with Coherence vector as given in Equation (16)

$E_{\text {diss }}=\frac{\hbar}{2} \int_{-L}^{L} \vec{\Gamma} \cdot \frac{d \vec{\lambda}}{d t} d t=\frac{\hbar}{2}\left([\vec{\Gamma} \cdot \vec{\lambda}] \frac{L}{-L}-\int_{-L}^{L} \vec{\lambda} \cdot \frac{d \vec{\Gamma}}{d t} d t\right)$.

The QCAPro tool uses these equations to provide different energies dissipated by the QCA circuits at three different tunneling energy levels which are $0.5 \mathrm{Ek}, 1.0 \mathrm{Ek}$ and $1.5 \mathrm{Ek}$. This tool also provides with graphical representation of energy dissipated by each cell in the design in the form of energy dissipation maps. Figure 23 shows the energy dissipation map for all designs at $0.5 \mathrm{Ek}$ level. The default temperature and other settings have been selected in the QCAPro tool to obtain these maps. The dark/black cells in these maps depicts the cells dissipating maximum energy in the circuit whereas the white cells are input cells which dissipate no energy. It is observed from the energy maps that only few cells in proposed designs are dark and hence the proposed FAS designs can be considered as energy efficient in nature.

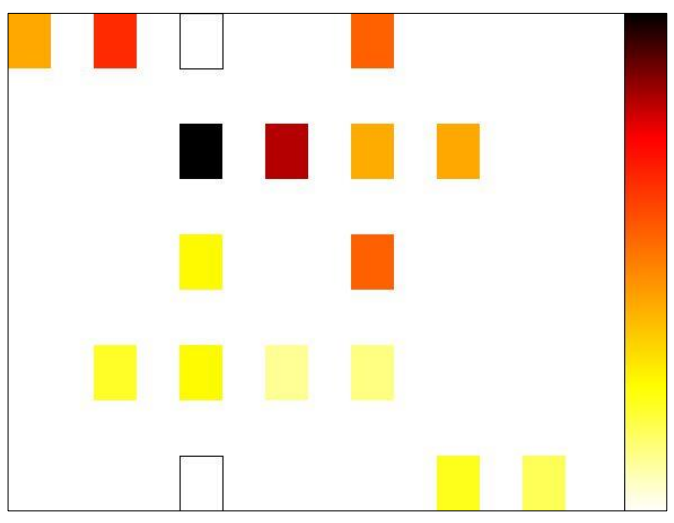

(a)

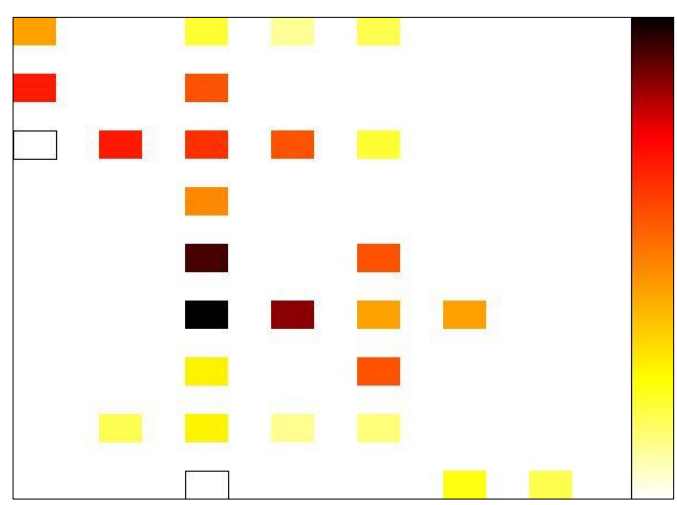

(c)

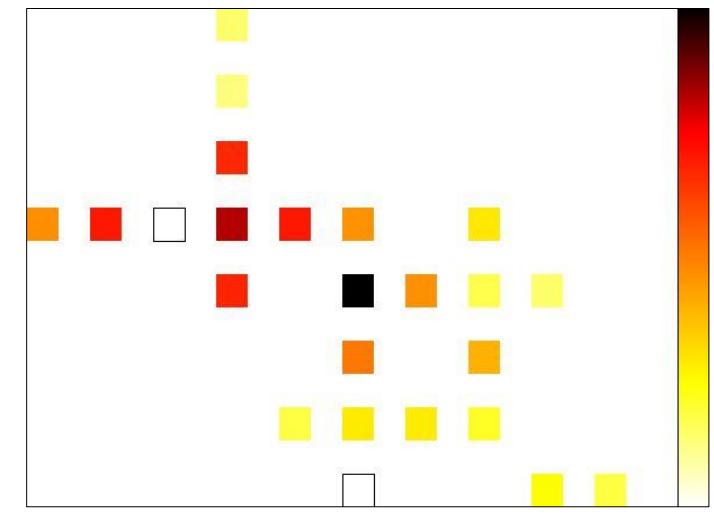

(b)

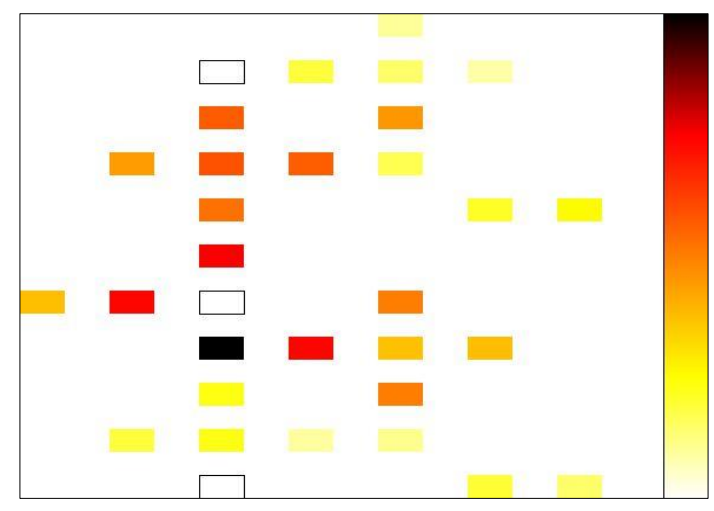

(d)

Figure 23. Energy dissipation map at 0.5 Ek energy level of proposed $F A S(a)$ design - 1, (b) design-2, (c) design-3 and (d) design - 4 at $2 \mathrm{~K}$ temp 


\section{DISCUSSION}

The efficiency of the proposed designs is evaluated by drawing a number of comparison tables. The comparison table showing the efficiency of the proposed design of FAS as compared to the existing design is given in Table 3.

Table 3. Comparison between previous and the proposed designs of FAS

\begin{tabular}{|c|c|c|c|c|c|}
\hline Structure & Cell count & $\begin{array}{c}\text { Cell Area } \\
\left(\boldsymbol{\mu ~ m}^{2}\right)\end{array}$ & $\begin{array}{c}\text { Total Area } \\
\left(\boldsymbol{\mu ~ m}^{2}\right)\end{array}$ & Latency & QCA Cost \\
\hline$[36]$ & 37 & 0.0148 & 0.0352 & 0.75 & 0.0198 \\
\hline $\begin{array}{c}\text { Proposed } \\
\text { Design-1 }\end{array}$ & 15 & 0.00486 & 0.011564 & 0.25 & 0.00723 \\
\hline $\begin{array}{c}\text { Proposed } \\
\text { Design-2 }\end{array}$ & 24 & 0.007776 & 0.031284 & 0.5 & 0.00782 \\
\hline $\begin{array}{c}\text { Proposed } \\
\text { Design-3 }\end{array}$ & 27 & 0.008748 & 0.024564 & 0.5 & 0.00614 \\
\hline $\begin{array}{c}\text { Proposed } \\
\text { Design-4 }\end{array}$ & 32 & 0.010368 & 0.030084 & 0.25 & 0.00188 \\
\hline
\end{tabular}

Here QCA cost is the product of total area and square of latency. In addition to this, parameter by parameter wise percentage improvement compared to existing design [36] is presented in Table 4. It is seen that the proposed FAS designs show an improvement ranging from $13.51 \%$ to $59.46 \%$ in terms of number of cells, $29.94 \%$ to $67.16 \%$ improvement in cell area, $11.12 \%$ to $67.14 \%$ improvement in total area, $33.33 \%$ to $66.67 \%$ improvement in latency and $60.51 \%$ to $90.51 \%$ improvement in terms of QCA circuit cost. It is also worth stating here that the proposed $3 \times 3$ Double Feynman Gate based Design- 4 of FAS has the ability to match two different fingerprints simultaneously and despite this additional feature, it achieves an improvement of $90.51 \%$ in terms of QCA circuit cost compared to the design proposed by [36] which uses $2 \times 2$ Feynman gate for its FAS design.

Table 4. Percentage improvement in proposed designs compared to design [36]

\begin{tabular}{|c|c|c|c|c|}
\hline Parameter & Design-1 & Design-2 & Design-3 & Design-4 \\
\hline Cell count & $59.46 \%$ & $35.14 \%$ & $27.03 \%$ & $13.51 \%$ \\
\hline $\begin{array}{c}\text { Cell Area } \\
\left(\mu \mathrm{m}^{2}\right)\end{array}$ & $67.16 \%$ & $47.46 \%$ & $40.89 \%$ & $29.94 \%$ \\
\hline $\begin{array}{c}\text { Total Area } \\
\left(\mu \mathrm{m}^{2}\right)\end{array}$ & $67.14 \%$ & $11.12 \%$ & $30.22 \%$ & $14.53 \%$ \\
\hline Latency & $66.67 \%$ & $33.33 \%$ & $33.33 \%$ & $66.67 \%$ \\
\hline QCA cost & $63.48 \%$ & $60.51 \%$ & $68.99 \%$ & $90.51 \%$ \\
\hline
\end{tabular}

A graphical correlation showing the energy effectiveness of the proposed FAS designs are shown in Figure 24. It is evident from the graphical comparison of energy dissipated by proposed designs that Design-4 dissipates the highest energy which is evident due to higher number of cells utilized in its design whereas, Design-1 dissipates the least energy. Depending upon the requirement of the user, any of the four proposed designs of Fingerprint Authentication Systems can used for secure nano-computing applications. 


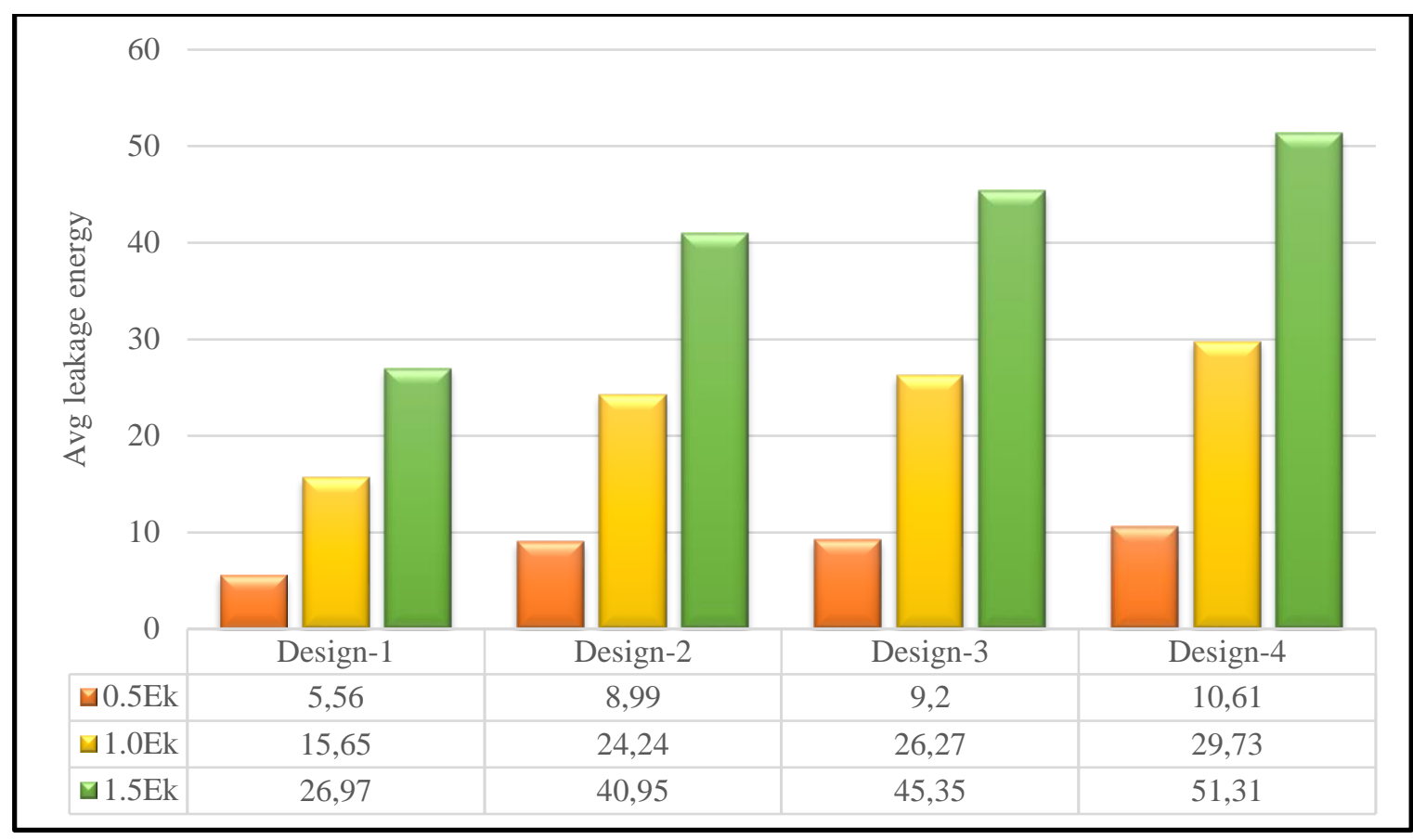

(a)

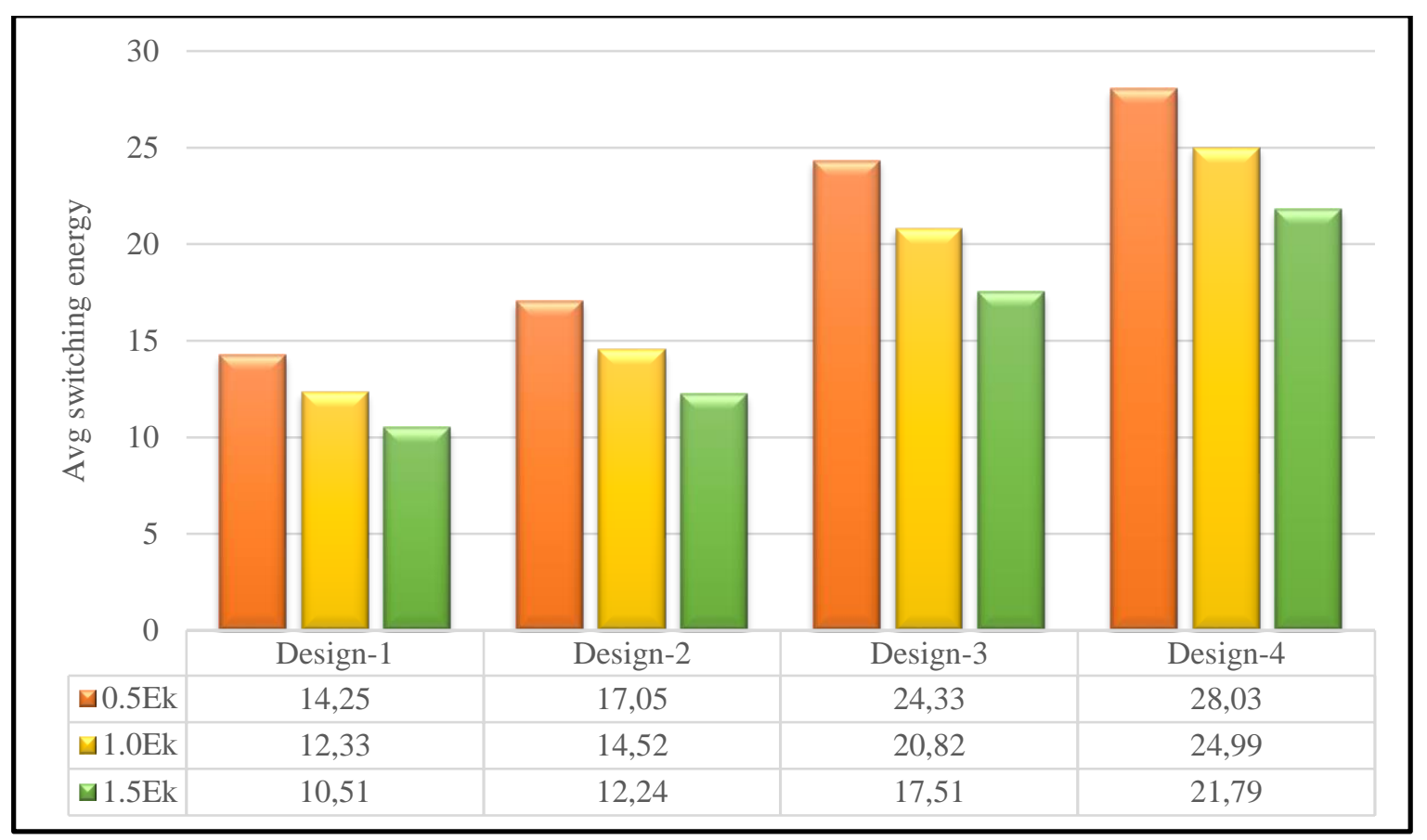

(b) 


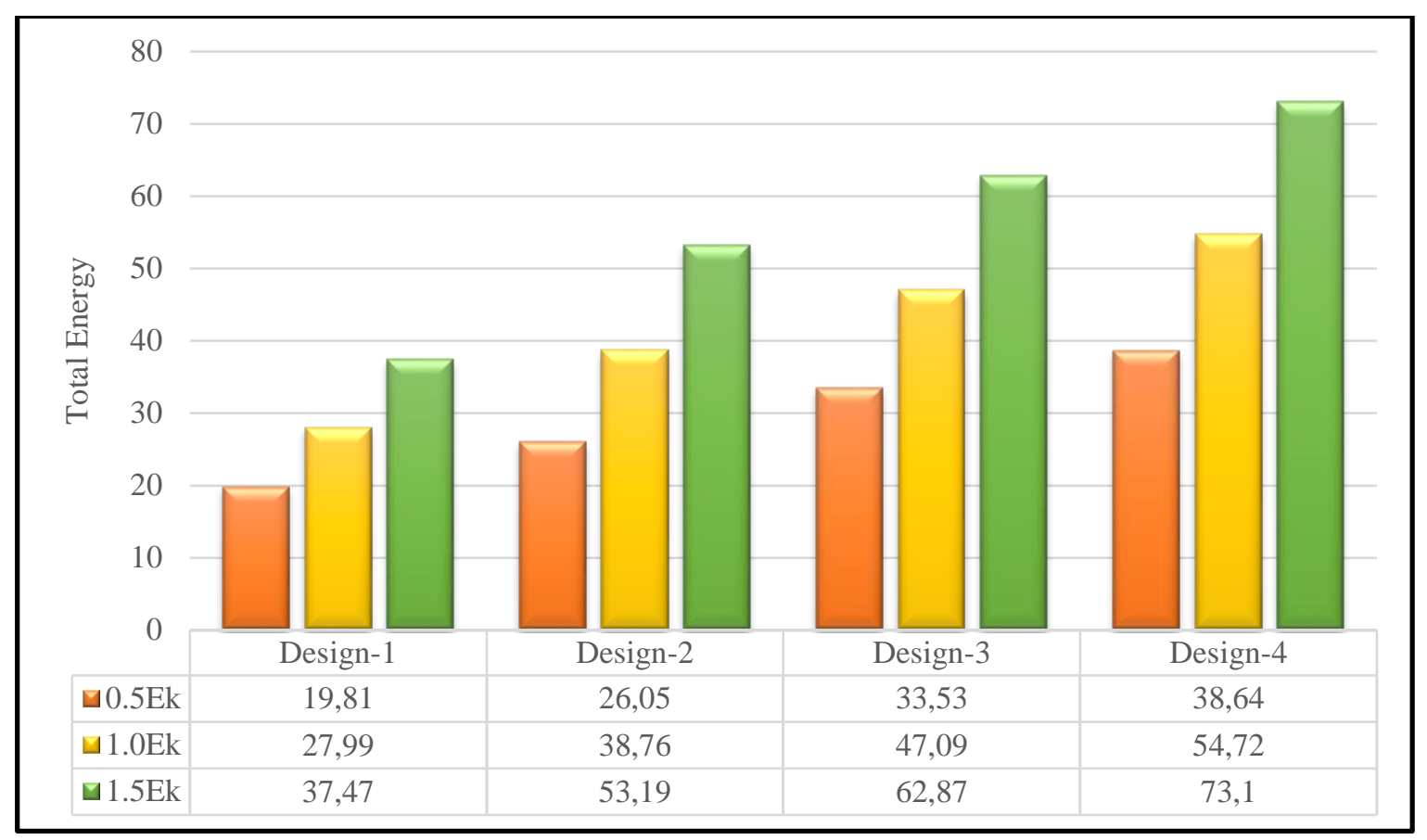

(c)

Figure 24. Comparison of (a) avg. leakage (b) avg. switching and (c) total energy (meV) dissipation of proposed FAS designs

\section{CONCLUSION}

This paper first presents the basic operating principle of a Fingerprint Authentication System (FAS). Its operation follows that of an XNOR gate which can be directly designed from an XOR gate. Hence, given the application and accuracy of the results required, a first of its kind fault tolerance analysis of five efficient XOR gate designs in the literature is presented. Based on the performance evaluation one of the design is selected and four fault tolerant designs of FAS are proposed which are based on different reversible gates. The simulation results are acquired utilizing the QCADesigner tool and the energy dissipation of proposed FAS designs is determined using the QCAPro tool. It is seen that the proposed designs are cost efficient and achieve improvements up to $59.46 \%$ in terms of number of cells, $67.16 \%$ improvement in cell area, $67.14 \%$ improvement in total area, $66.67 \%$ improvement in latency and $90.51 \%$ improvement in terms of circuit cost compared to the design. Additionally, based on the energy dissipation analysis for different kink energy levels, it is seen that the presented FAS designs dissipate very low energy which make them suitable for application in different ultra-low power nano-computing applications.

\section{CONFLICTS OF INTEREST}

No conflict of interest was declared by the authors.

\section{FUNDING INFORMATION}

The authors would like to thank National Project Implementation Unit (NPIU), a unit of Ministry of Education, Government of India and The World Bank, for providing financial assistance to carry out this research under TEQIP-III Collaborative Research Scheme (CRS) with sanction number: 1-5736612144. 


\section{REFERENCES}

[1] Lent, C. S., Tougaw, P., Porod, W., and Bernstein, G. H., "Quantum cellular automata", Nanotechnology, 4(1): 49, (1993).

[2] Smith, C. G., "Computation without current", Science, 284(5412): 274-274, (1999).

[3] Bilal, B., Ahmed, S., Kakkar, V., "Quantum Dot Cellular Automata: A New Paradigm for Digital Design", International Journal of Nanoelectronics and Materials, 11(1): 87-98, (2018).

[4] Bilal, B., Ahmed, S., Kakkar, V., "Modular adder designs using optimal reversible and fault tolerant gates in field-coupled QCA nanocomputing", International Journal of Theoretical Physics, 57(5): 1356-1375, (2018).

[5] Ahmad, F., Ahmed, S., Kakkar, V., Bhat, G. M., Bahar, A. N., Wani, S., "Modular Design of UltraEfficient Reversible Full Adder-Subtractor in QCA with Power Dissipation Analysis", International Journal of Theoretical Physics, 57 (9): 2863-2880, (2018).

[6] Bilal, B., Ahmed, S., Kakkar, V., "Multifunction reversbile logic gate: Logic synthesis and design implementation in QCA", IEEE International Conference on Computing, Communication and Automation (ICCCA), Greater Noida, India, 1385-1390, (2017).

[7] Nafees, N., Manzoor, I., Baba, M. I., Bhat, S. M., Puri, V., Ahmed, S., "Modeling and Logic Synthesis of Multifunctional and Universal $3 \times 3$ Reversible Gate for Nanoscale Applications", In: Singh Tomar G., Chaudhari N., Barbosa J., Aghwariya M. (eds) International Conference on Intelligent Computing and Smart Communication, Algorithms for Intelligent Systems, Springer, Singapore, 1423-1431, (2019).

[8] Manzoor, I., Nafees, N., Baba, M. I., Bhat, S. M., Puri, V., Ahmed, S., "Logic Design and Modeling of an Ultraefficient $3 \times 3$ Reversible Gate for Nanoscale Applications", In: Singh Tomar G., Chaudhari N., Barbosa J., Aghwariya M. (eds) International Conference on Intelligent Computing and Smart Communication, Algorithms for Intelligent Systems, Springer, Singapore, 1433-1442, (2019).

[9] Bilal, B., Ahmed, S., Kakkar, V., "Optimal realization of universality of peres gate using explicit interaction of cells in quantum dot cellular automata nanotechnology", International Journal of Intelligent Systems and Applications, 9(6): 75-84, (2017).

[10] Raj, M., Ahmed, S., Gopalakrishnan, L., "Subtractor circuits using different wire crossing techniques in quantum-dot cellular automata", Journal of Nanophotonics, 14(2): 026007, (2020).

[11] Ahmed, S., Baba, M. I., Bhat, S. M., Manzoor, I., Nafees, N., Ko, S.-B.., "Design of reversible universal and multifunctional gate-based 1-bit full adder and full subtractor in quantum-dot cellular automata nanocomputing", Journal of Nanophotonics, 14(3): 036002, (2020).

[12] Bhat, S. M., Ahmed, S., "Design of Ultra-Efficient Reversible Gate Based 1-bit Full Adder in QCA with Power Dissipation Analysis", International Journal of Theoretical Physics, 58(12): 4042-4063, (2019).

[13] Roohi, A., Zand, R., Angizi, S., DeMara, R. F., "A parity-preserving reversible QCA gate with self-checking cascadable resiliency", IEEE Transactions on Emerging Topics in Computing, 6(4): 450-459, (2016).

[14] Fredkin, E.,Toffoli, T., "Conservative logic", International Journal of Theoretical Physics, 21(3-4): 219-253, (1982). 
[15] Ma, X., Huang, J., Metra, C., Lombardi, F., "Reversible and testable circuits for molecular QCA design", In: Tehranipoor M. (eds), Emerging Nanotechnologies. Frontiers in Electronic Testing, vol 37, Springer, Boston, MA, 157-202, (2008).

[16] Sen, B., Saran, D., Saha, M., Sikdar, B. K., "Synthesis of reversible universal logic around QCA with online testability", IEEE International Symposium on Electronic System Design, 236-241, (2011).

[17] Moharrami, E., Navimipour, N. J., "Designing nanoscale counter using reversible gate based on quantum-dot cellular automata", International Journal of Theoretical Physics, 57(4): 1060-1081, (2018).

[18] Das, J. C., De, D., "QCA based design of Polar encoder circuit for nano communication network", Nano Communication Networks, 18: 82-92, (2018).

[19] Agrawal, P., Sinha, S., Misra, N. K., Wairya, S., "Design of Quantum Dot Cellular Automata Based Parity Generator and Checker with Minimum Clocks and Latency", International Journal of Modern Education and Computer Science, 8(8): 11-20, (2016).

[20] Bahar, A. N., Uddin, M. S., Abdullah-Al-Shafi, M., Bhuiyan, M. M. R., Ahmed, K., "Designing efficient QCA even parity generator circuits with power dissipation analysis", Alexandria Engineering Journal, 57(4): 2475-2484, (2018).

[21] Das, J. C., De, D., Sadhu, T., "A novel low power nanoscale reversible decoder using quantum-dot cellular automata for nanocommunication", 3rd IEEE International Conference on Devices, Circuits and Systems (ICDCS), 220-224, (2016).

[22] Singh, R., Sharma, D. K., "Fault Tolerant Reversible Gate Based Sequential Quantum Dot Cellular Automata Circuits: Design and Contemplation", Journal of Nanoelectronics and Optoelectronics, 15(3): 331-344, (2020).

[23] Abutaleb, M., "Robust and efficient quantum-dot cellular automata synchronous counters", Microelectronics Journal, 61: 6-14, (2017).

[24] Divshali, M. N., Rezai, A., Hamidpour, S. S. F., "Design of Novel Coplanar Counter Circuit in Quantum Dot Cellular Automata Technology", International Journal of Theoretical Physics, 58(8): 2677-2691, (2019).

[25] Jeon, J.-C., "Low-complexity QCA universal shift register design using multiplexer and D flip-flop based on electronic correlations", The Journal of Supercomputing, 76(8): 6438-6452, (2020).

[26] Sadhu, A., Das, K., De, D., Kanjilal, M. R., "Area-Delay-Energy aware SRAM memory cell and $\mathrm{M} \times \mathrm{N}$ parallel read/write memory array design for quantum dot cellular automata", Microprocessors and Microsystems, 72: 102944, (2020).

[27] Fam, S. R., Navimipour, N. J., "Design of a loop-based random access memory based on the nanoscale quantum dot cellular automata", Photonic Network Communications, 37(1): 120-130, (2019).

[28] Mubarakali, A., Ramakrishnan, J., Mavaluru, D., Elsir, A., Elsier, O., Wakil, K., "A new efficient design for random access memory based on quantum dot cellular automata nanotechnology", Nano Communication Networks, 21: 100252, (2019).

[29] Heikalabad, S. R., Navin, A. H., Hosseinzadeh, M., "Content addressable memory cell in quantumdot cellular automata", Microelectronic Engineering, 163: 140-150, (2016). 
[30] Amlani, I., Orlov, A. O., Toth, G., Bernstein, G. H., Lent, C. S., Snider, G. L., "Digital logic gate using quantum-dot cellular automata", Science, 284(5412): 289-291, (1999).

[31] Niemier, M. T., Kogge, P. M., "Logic in wire: using quantum dots to implement a microprocessor", 6th IEEE International Conference on Electronics, Circuits and Systems (Cat. No. 99EX357), 1211-1215, (1999).

[32] Frost, S. E., Rodrigues, A. F., Janiszewski, A. W., Rausch, R. T., Kogge, P. M., "Memory in motion: A study of storage structures in QCA", First Workshop on Non-Silicon Computing, 1-8, (2002).

[33] Bilal, B., Ahmed, S., Kakkar, V., "An insight into beyond CMOS next generation computing using quantum-dot cellular automata nanotechnology", International Journal of Engineering and Manufacturing, 8(1): 25-37, (2018).

[34] Roohi, A., DeMara, R. F., Khoshavi, N., "Design and evaluation of an ultra-area-efficient faulttolerant QCA full adder", Microelectronics Journal, 46(6): 531-542, (2015).

[35] Ahmed, S., Bhat, S. M., Ko, S. B., "Design of reversible gate-based fingerprint authentication system in quantum-dot cellular automata for secure nanocomputing", In: Singh P. K., Singh Y., Kolekar, M. H., Kar, A. K., Chhabra J. K., Sen A. (eds) International Conference on Recent Innovations in Computing, Lecture Notes in Electrical Engineering, 701, Springer, Singapore, 729740, (2020).

[36] Debnath, B., Das, J. C., De, D., "Fingerprint authentication using QCA technology", IEEE Conference on Devices for Integrated Circuit (DevIC), 125-130, (2017).

[37] Majeed, A. H., Zainal, M. S. B., Alkaldy, E., Nor, D. M., "Full Adder Circuit Design with Novel Lower Complexity XOR Gate in QCA Technology", Transactions on Electrical and Electronic Materials, 21: 198-207, (2020).

[38] Chabi, A. M., Roohi, A., DeMara, R. F., Angizi, S., Navi, K., Khademolhosseini, H., "Costefficient QCA reversible combinational circuits based on a new reversible gate", 18th IEEE CSI International Symposium on Computer Architecture and Digital Systems (CADS), 1-6, (2015).

[39] Bahar, A. N., Waheed, S., Hossain, N., Asaduzzaman, M., "A novel 3-input XOR function implementation in quantum dot-cellular automata with energy dissipation analysis", Alexandria Engineering Journal, 57(2): 729-738, (2018).

[40] Ahmed, S., Naz, S. F "Design of quantum dot cellular automata based fault tolerant convolution encoders for secure nanocomputing", International Journal of Quantum Information, 18(6): 2050032, (2020).

[41] Walus, K., Dysart, T. J., Jullien, G. A., Budiman, R. A., “QCADesigner: a rapid design \& simulation tool for quantum-dot cellular automata", IEEE Transaction on Nanotechnology, 3(1): 26-31, (2004).

[42] Feynman, R. P., "Quantum mechanical computers", Foundations of Physics, 16(6): 507-532, (1986).

[43] Nagamani, A., Jayashree, H., Bhagyalakshmi, H., "Novel low power comparator design using reversible logic gates", Indian Journal of Computer Science and Engineering, 2(4): 566-574, (2011). 
[44] Toffoli, T., "Reversible computing", International Colloquium on Automata, Languages and Programming, Springer, 632-644, (1980).

[45] Parhami, B., "Fault-tolerant reversible circuits", Fortieth IEEE Asilomar Conference on Signals, Systems and Computers, 1726-1729, (2006).

[46] Srivastava, S., Asthana, A., Bhanja, S., Sarkar, S., "QCAPro-an error-power estimation tool for QCA circuit design", IEEE International Symposium of Circuits and Systems (ISCAS), 2377-2380, (2011).

[47] Srivastava, S., Sarkar, S., Bhanja, S., "Estimation of upper bound of power dissipation in QCA circuits", IEEE Transactions on Nanotechnology, 8(1): 116-127, (2008).

[48] Timler, J., Lent, C. S., "Power gain and dissipation in quantum-dot cellular automata", Journal of Applied Physics, 91(2): 823-831, (2002). 